Document downloaded from:

http://hdl.handle.net/10251/37967

This paper must be cited as:

Rodríguez Álvarez, MJ.; Soriano Asensi, A.; Iborra Carreres, A.; Sánchez Martínez, F.; González Martínez, AJ.; Conde, P.; Hernández Hernández, L.... (2013). Expectation maximization (EM) algorithms using polar symmetriesfor computed tomography(CT) image reconstruction. Computers in Biology and Medicine. 43(8):1053-1061. doi:10.1016/j.compbiomed.2013.04.015.

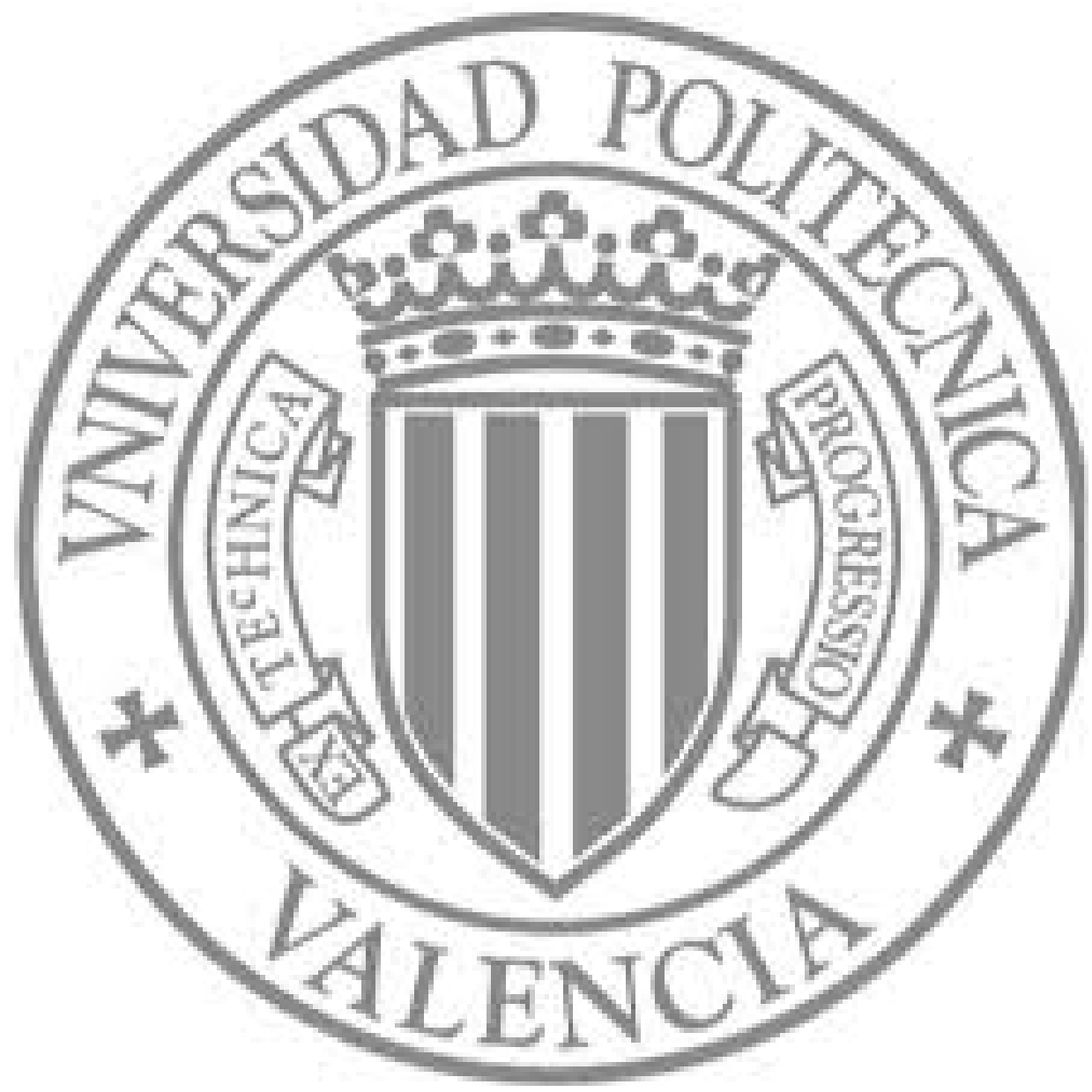

The final publication is available at

http://dx.doi.org/10.1016/j.compbiomed.2013.04.015

Copyright Elsevier 


\title{
Expectation maximization (EM) algorithms using polar symmetries for computed tomography (CT) image reconstruction.
}

\author{
M.J. Rodríguez-Alvarez ${ }^{1}$, A. Soriano ${ }^{1}$, A. Iborra ${ }^{1}$, F. Sánchez ${ }^{1}$, A.J. \\ González $^{1}$, P. Conde $^{1}$, L. Hernández ${ }^{1}$, L. Moliner ${ }^{1}$, A. Orero ${ }^{1}$, L.F. Vidal and \\ J.M. Benlloch ${ }^{1}$ \\ ${ }^{1}$ Instituto de Instrumentación para Imagen Molecular (I3M), Centro mixto \\ CSIC-Universitat Politécnica de Valencia-CIEMAT, Camino de Vera s/n E-46022 \\ Valencia (Spain)
}

\begin{abstract}
We suggest a symmetric-polar pixellation scheme which makes possible a reduction of the computational cost for expectation maximization (EM) iterative algorithms. The proposed symmetric-polar pixellation allows us to deal with $3 \mathrm{D}$ images as a whole problem without dividing the $3 \mathrm{D}$ problem into $2 \mathrm{D}$ slices approach. Performance evaluation of each approach in terms of stability and image quality are presented. Exhaustive comparisons between all approaches were conducted in a $2 \mathrm{D}$ based image reconstruction model. From these 2D approaches, that showing the best performances was finally implemented and evaluated in a 3D based image reconstruction model. Comparison to 3D images reconstructed with FBP is also presented. Although the algorithm is presented in the context of computed tomography (CT) image reconstruction, it can be applied to any other tomographic technique as well, due to the fact that the only requirement is a scanning geometry involving measurements of an object under different projection angles. Real data has been acquired with a small animal (CT) scanner to verify the proposed mathematical description of the CT
\end{abstract}

Email address: mjrodri@i3m.upv.es (M.J. Rodríguez-Alvarez ${ }^{1}$ ) 
system.

Keywords: Computed tomography, polar geometry, MLEM, FBP, OSEM, 3D images. 


\section{Introduction}

Image reconstruction in computed tomography (CT) has been dominated by analytical methods like filtered backprojection (FBP) [1] because these methods produce images of a reasonable quality with low cost in terms of computing time. Advances in computer science enable the use of reconstruction techniques based on iterative methods [2], [3] as an alternative to FBP, although the time needed for image reconstruction increases considerably [4].

Iterative methods allow a detailed mathematical description of the physical processes involved in tomographic systems, such as the attenuation and scatter of photons in the body under study [5]. As a consequence of the improvement of the image quality, less patient dose is needed during CT examination [6], [7]. Promising results obtained at the Mayo Clinic in Arizona reported a $63 \%$ reduction in dose received by the patient when iterative reconstruction methods were implemented instead of FBP [8].

Iterative methods consider the image reconstruction problem as a discrete linear system, where the mathematical description of the tomographic system is done through the so-called weights matrix. Noise in the iterative images can be reduced by about $60 \%$ compared to the FBP results without compromising spatial resolution [9]. Tomographic imaging systems have been greatly improved in the last few years [10] whereas several methods have been proposed to accelerate the convergence of iterative methods [11-15]. Great improvements have been obtained by reducing the system matrix size taking into account the geometry of the system [16], and consequently reducing the computational complexity. Despite the complexity of the problem, several methods have been proposed to improve the calculation of the weighting factor values [17], [18].

Small animal CT imaging has gained considerably importance as a method for preclinical studies in the last decades [19]. Due to the high resolution needed 
when imaging small animals (typically $0.1 \mathrm{~mm}$ ) accurate image reconstruction algorithms are required. In this work, we focus on the development of a CT system matrix description that allows us to use the maximum likelihood expectation maximization (MLEM) and ordered subsets expectation maximization (OSEM) algorithms reducing the computational complexity without decreasing the quality and stability of images.

The final goal in this paper is to drastically reduce computational cost (200 times at least) allowing us to reconstruct three dimensional (3D) CT images without losing quality.

Performance evaluation of the various approaches proposed in this work was conducted with real data from a small animal micro CT. A dedicated phantom with synthetic materials modelling different tissue densities has been designed and manufactured for this study. The FBP algorithm has been implemented to compare 3D iterative images against the customarily used algorithm for $\mathrm{CT}$ image reconstruction.

\section{Iterative reconstruction}

The iterative image reconstruction can be regarded as a statistical estimation problem. The expectation-maximization (EM) algorithms can be used to compute the estimated maximum-likelihood when the projection data are not equally spaced or the observation can be viewed as an incomplete data set [9], [11] and [20]. The MLEM algorithm [2] has attracted considerable interest in the area of tomographic image reconstruction, as it produces high quality images. However these methods are computationally intensive and may suffer from a slow convergence rate. To overcome this, the OSEM algorithm was proposed in 1994 [3]. The OSEM algorithm is a modified version of MLEM algorithm that groups projections into subsets of angles. The image is updated as many 
times as the number of subsets, proportionally accelerating convergence for each iteration.

Several pixellation configurations of the field of view (FOV) have been proposed in order to decrease the computational cost and to improve the image reconstruction quality using alternative models such as region of interest [21], blobs [22-24], natural pixels and strip functions [25]. In order to take full advantage of the symmetry of the scanner geometry, we proposed to use polar discretization to arrange the pixels in the FOV [26], see Fig. 1(a). The FOV is split into as many sectors as the number of projections and each sector is subdivided into voxels. This division of the FOV allows us to recycle the weights between consecutive projections, since the system matrix of the CT is constructed as a block circulant matrix [27]. Since more than one hundred projections are usually required to obtain a $\mathrm{CT}$ image, the polar description cuts down the time and storage requirements of the weights matrix. The authors have recently studied the 2D approach [28], showing that polar pixel-based weights matrices allow a highly efficient implementation of the 2D MLEM. Three different methods of obtaining the weights matrix using polar discretization are presented and compared in the 2D CT case. The best $2 \mathrm{D}$ weights matrix model is extended to 3D, which allows full 3D iterative reconstruction in order to obtain volumetric images.

To display images that are arranged in a polar configuration, it is necessary to design an additional procedure to represent the information in a cartesian grid. The transformation is based on an interpolation that converts the reconstructed polar pixels image to another in square pixels and places each polar pixel on a Cartesian-grid thin enough to keep the resolution of the original polar image [28].

\section{Preferred position for figure 1}




\subsection{Weights matrix}

The mathematical description of the CT scanner in iterative reconstruction algorithms is contained in a weights matrix. The element $w_{i j}$ of the weights matrix represents the contribution of each voxel $j$ to the attenuation of beam $i$. The way the $w_{i j}$ are defined, for each pixel (voxel in 3D), strongly influences the quality of the reconstructed image obtained after the iterative process.

The number of $w_{i j}$ elements required to consider all the contributions of a voxel in all projections is too large to be stored and managed in a standard upto-date computer. Consequently, it is necessary to reduce as much as possible the number of $w_{i j}$ elements to be computed. Acquired data can be considered as a sequence of measurements taken from each one of the projections of the scanner, see Fig. 1(a). Therefore, the geometry of the scanner with a polar pixellation has as many symmetries as projections have been taken, see Fig. 1(b). This symmetry may be exploited to reduce the number of weighting factors using polar geometry [26], [28]. Due to the polar symmetry, only the elements related to the first projection have to be calculated while the matrix elements of the remaining projections can be obtained through rotation of the first projection. Three methods have been chosen to calculate the weights matrix elements in 2D nearest neighbour, Joseph, and intersected area. The objective of this comparison is to study how much detailed matrix weight is required to achieve an optimal image quality, since the size of the system matrix required to describe a cone-beam (3D) scanner is 100 to 1000 times larger than the one used to describe a fan-beam (2D).

\subsection{1. $2 D$ weights matrix}

Nearest neighbour. It considers that each pixel only contributes to the attenuation measured in the closest beam.

Joseph's Method (Joseph 1982). It assumes that each pixel contributes to 
the attenuation measured in its two surrounding beams. The weighting factor decreases linearly with the relative distance of the pixel respect to each beam [29].

Intersected Area. For this strategy a beam is defined as the area delimited by the X-ray source and the two lateral limits of each detector element. The pixels are polar sectors [28] and $w_{i j}$ is defined as the intersected area between pixel $j$ and beam $i$.

\subsection{2. $3 D$ weights matrix}

The 3D grid is constructed inserting a third cylindrical coordinate to turn the 2D polar pixels into a 3D voxel, see Fig. 1(b) and Fig. 2. As it will be described below, the "intersected area" procedure described above for 2D has produced the best results. For that reason, we have extended this procedure to $3 \mathrm{D}$ in order to determine the weight element $w_{i j}$. Now, each beam is considered as a pyramid defined by each pixel in the detector and the focal spot, see Fig. 2. The contribution of each voxel corresponds to the intersected volume between the voxel and the beam.

\section{Preferred position for figure 2}

The volume is calculated in two steps. First, the area intersected between the top cover of the voxel and the two lateral sides of the beam is calculated. Points P1, P2, P3 and P4, see Fig. 3(a), are calculated and therefore, the area between the points. The geometric center $\mathrm{C}$ of the area is also calculated.

\section{Preferred position for figure 3}

Figure is not to scale, focal distance between detectors and X-ray source is diminished in order to enable us to see the intersected area. Second, the length of the height intersected between the voxel and each beam at the midpoint $\mathrm{C}$, see Fig. 3(b), is calculated in such a way that the volume intersected by the beam $\mathrm{B} 1$ through the pixel is the area previously calculated multiplied by height $\mathrm{h} 1$, 
the volume intersected by the beam B2 is the same area multiplied by the height h2 and so on for beam B3 and height h3.

\section{Materials and methods}

\subsection{Experimental device}

Experimental data were acquired with a small animal micro CT scanner [30]. It mounts a microfocus X-ray tube with a $35 \mu \mathrm{m}$ focal spot size. Although variable voltage settings from 0 to $50 \mathrm{kV}$ were available, the tube voltage was fixed to $40 \mathrm{kV}$ in all the acquisitions performed for this study. A $0.5 \mathrm{~mm}$ aluminum filter was used to attenuate photons with energies below $20 \mathrm{kV}$ that would increase the dose in peripheral organs while no contributing to image quality [19], making it possible for us to simulate preclinical CT conditions as well. The X-ray detector is a CsI scintillator flat panel with a $2400 \times 2400$ pixels array of $50 \times 50 \mu \mathrm{m}$, totalizing a $120 \times 120 \mathrm{~mm}$ detection area. The $\mathrm{X}$-ray tube and detector are mounted in a cone-beam configuration so that the scanner has a FOV of $80 \mathrm{~mm}$ in diameter.

A cylindrical phantom of polymethylmethacrylate (PMMA) $50 \mathrm{~mm}$ height and $55 \mathrm{~mm}$ in diameter was used, see Fig. 4. Five holes of $8 \mathrm{~mm}$ in diameter were axially drilled at $16 \mathrm{~mm}$ off the axis. Three of the holes were filled with $8 \mathrm{~mm}$ in diameter inserts of polyethylene (PE), polyoxymethylene (POM) and polytetrafluoroethylene (PTFE), which model adipose tissue, organs tissue and soft bone respectively. A PMMA insert was placed in the fourth hole, and was used to align the phantom in the scanner. The remaining hole was left empty to model an air region inside the body.

\section{Preferred position for figure 4}

Data acquisitions consisted of 400 projections of the described phantom, although 180 projections were enough to reconstruct the phantom considered in 
this study. However, the increase in the number of projections allowed a wider range in the number of subsets. 1, 10, 20, 40 and 100 subsets were considered during the reconstruction in this study. Increasing the number of projections also improves the accuracy of the reconstructed image reducing the influence of noise on experimental data into the obtained results.

The dose [31], [32] per projection was $0.8 \mathrm{mGy}$, producing a total irradiation of $320 \mathrm{mGy}$ in the case of 400 projections.

The experimental data set used for the comparison of algorithms in 2D was obtained by selecting the central slice of each projection. This facilitates the comparison among results obtained in 2D and 3D.

\subsection{Image quality indicators}

The performance evaluation of the various weights matrices considered in this work was based on the monitoring of three quality indicators during the iterative process. These parameters are evaluated in five cylindrical regions of interest (RoI) defined in the center of each insert. The RoIs were $4.5 \mathrm{~mm}$ in diameter, and $20 \mathrm{~mm}$ long in the 3D images. Parameters were monitored after each image update so that it was possible to analyze the evolution of the values in each RoI to study the convergence process of the algorithm. Although customary image reconstructions consist of no more than 50 updates, the iterative process was extended to 500 updates in order to verify the stability of the new polar arrangement of pixels and convergence of each approach considered. These were the quality indicators considered:

- $C T$ number $(C T)$ permits to study how fast each RoI converges on the final value that will be appreciated in the reconstructed image.

$$
C T=\frac{1}{N_{R}} \sum_{i=1}^{N_{R}} x_{i},
$$


where $x_{i}$ are the values of the pixels in the RoI, and $N_{R}$ the number of pixels.

- Coefficient of variation $(C V)$ is an estimator of the average difference among the pixel values in the RoI. The $C V$ is divided by the average $C T$ number in the RoI in order to get a dimensionless estimator of the noise in the RoI.

$$
C V=\frac{1}{C T} \sqrt{\frac{1}{N_{R}-1} \sum_{i=1}^{N_{R}}\left(x_{i}-C T\right)^{2}} 100,
$$

- Contrast Recovery Coefficient (CRC) quantifies how precisely the image reconstruction reproduces the relationships among $\mathrm{CT}$ number.

$$
C R C=100 \frac{\left(C T_{R o I}^{e x p} / C T_{B a c k}^{e x p}\right)-1}{\left(C T_{R o I} / C T_{B a c k}\right)-1}
$$

where $C T_{R o I}^{e x p}$ and $C T_{B a c k}^{e x p}$ are the average of the obtained $C T$ values in the RoI and in the PMMA background, while $C T_{R o I}$ and $C T_{B a c k}$ are the $\mathrm{CT}$ numbers of the materials in the insert and background obtained from [33], respectively.

\section{Results}

Detailed performance comparison among the approaches considered in this work has been conducted in 2D. Once an exhaustive 2D evaluation was done, the approach with the best performance was extended to 3D. Since similar behavior, in terms of convergence rate and stability, has been observed in all RoIs (see section 3.1), only the results corresponding to PE are shown in this paper.

\subsection{Comparison between $2 D$ methods}

The comparison among the approaches considered to calculate the weights matrix elements was done by analyzing the evolution of the CT number (Fig. 5), 
and the $C V$ (Fig. 6), during the iterative process in each RoI. All the methods were compared using 1, 10, 20 and 100 subsets per iteration.

\section{Preferred position for figure 5}

\section{Preferred position for figure 6}

When 1 to 20 subsets were considered for the $C T$ number, the iterative process converged on the final value after roughly 50 updates, with little variations, see Fig. 5. Differences arose when 100 subsets were considered. Instead of remaining stable after the initial convergence, an undesired oscillation was observed when 100 subsets were used. For the intersected area approach, the oscillation occurred around the value at which the image converges when 1 , 10 or 20 subsets were used. In the remaining two approaches the $C T$ number oscillated around different values leading to an incorrect solution.

Although $C T$ number nearly converged on the same value in all approaches, except when 100 subsets were utilized, strong differences were observed in the noise $(C V)$ obtained in the various approaches considered, see Fig. 6. $C V$ values above $22 \%$ were measured when the nearest neighbour approach was considered. In the Joseph's method it fell to $6 \%$ and using the intersected area approach, noise levels displayed in the image further improved to $4 \%$. Slight variations in $C V$ were observed when considering different number of subsets. Transversal slices of the reconstructed object are shown in Fig. 7. Strong artifacts were obtained for the nearest neighbour approach. Artifacts diminished considerably for Joseph's method, although slight artifacts were still visible. No evidences of ring artifacts were observed in Fig. 7 when the weights matrix elements were calculated using the intersected area approach.

\section{Preferred position for figure 7}

Therefore the approach with the best $2 \mathrm{D}$ performance, i.e. intersected area was extended to 3D. 


\subsection{Effect of the number of subsets}

The number of subsets considered during the iterative process influences the convergence of the reconstructed image. The convergence of the iterative process in the intersected area approach when 1, 10, 20, 40 and 100 subsets were considered is shown in Fig. 8 (2D) and in Fig. 9 (3D). Similar results to those shown in 2D Fig. 8 were observed in 3D Fig. 9. When less than 20 subsets were used, $C T$ number in the RoI rapidly converged during the 50 initial updates and remained stable up to the end of the iterative process. The same effect was observed in the $C R C$ which tended to $80 \%$.

\section{Preferred position for figure 8}

\section{Preferred position for figure 9}

As expected, $C V$ increased during the iterative process. However, $C V$ values below $5 \%$ were obtained with $2 \mathrm{D}$ images and slightly smaller values below $4 \%$ with 3D reconstructed images.

Taking into account the obtained results in Fig. 8 and Fig. 9, 20 subsets per iteration were used in all the remaining reconstructions performed for this study.

\subsection{Quantitative comparison between system matrices}

In order to make a 3D polar and cartesian matrices comparison by using the same computer (6 GB RAM) we had to simplify the calculation of the cartesian matrix. The comparison between both matrices at voxel size of $0.4 \times 0.4 \times 0.4 \mathrm{~mm}^{3}$ and $0.8 \times 0.8 \times 0.8 \mathrm{~mm}^{3}$ is shown in Tab. 1.

\section{Preferred position for table 1}

Polar matrix generation times are between 5 seconds and 12 seconds, depending only on the voxel sizes. In the case of cartesian matrix, generation times are between 88 seconds and 630 seconds, depending not only of the voxel size, but also on the number of projections. 
The polar matrix size did not change significatively with the number of projection angles. However, the size of the cartesian matrix increased linearly with the number of projection angles. For the case of $0.8 \times 0.8 \times 0.8 \mathrm{~mm}^{3}$ voxels, the smallest matrix, similar reconstruction times were measured with polar and cartesian matrices.

\subsection{Comparison of results for FBP and OSEM}

3D reconstructed images with FBP and MLEM are shown in Fig. 10. When comparing reconstructed images with the same voxel size of $0.8 \mathrm{~mm}$, OSEM exhibits a superior quality. If the voxel size in the FBP reconstruction is reduced to $0.4 \mathrm{~mm}$ of the image quality improves, being comparable to that in obtained with iterative methods, see Fig. 10(c).

\section{Preferred position for table 2}

\section{Preferred position for table 3}

\section{Preferred position for figure 10}

A quantitative comparison between FBP and iterative method OSEM is shown in Tab. 2 and Tab. 3. The iterative reconstruction procedure consists of 5 iterations with the OSEM algorithm and 20 subsets. Results in Tab. 2 reflect an increase of the noise levels in all RoIs when the number of projections diminishes.

\section{Discussion}

In the $2 \mathrm{D}$ case, the intersected area approach produces reconstructed images with higher quality when compared with the other two considered approaches (see Fig. 5 and Fig. 6), being less sensitive to produce ring artifacts (see Fig. 7). For those reasons, the intersected area approach was chosen for the 3D study.

In spite of using the same projection data in $2 \mathrm{D}$ and $3 \mathrm{D}$ reconstructions, slight differences are observed between Fig. 8 and Fig. 9. Results are slightly 
different because 2D results of Fig. 8 were calculated using 2D RoIs and 3D results of Fig. 9 were calculated using cylindrical volume of interest (VoIs).

A detailed analysis of the effect of the number of subsets used over the image quality was carried out in 2D and 3D. In all three approaches considered to calculate the weights matrix, a decrease in image quality was appreciated as the number of subsets increases. Common experience with OSEM has shown that the main advantage is a gain factor in processing time roughly equal to the number of subsets. But there is a limit for this effect. The OSEM algorithm is more likely to diverge when few projections per subset are considered [34], [35]. So, there is an optimal number of subsets. In our case about 20 subsets is an optimal number, it can be observed that results slightly worse are obtained for 40 subsets and the worst results are obtained for 100 subsets. Therefore, a satisfactory concurrence between theoretical and image reconstructed values is observed in Fig. 8 and Fig. 9.

The main advantage of the CT system matrix when using the polar scheme is its reduced size when compared with the cartesian matrix size (Tab. 1). Moreover, the polar matrix was a great deal smaller and could stay in RAM memory in a standard computer, thus allowing faster access speed for $0.4 \times 0.4 \times 0.4 \mathrm{~mm}^{3}$ voxels. Polar matrix makes possible to reconstruct 3D images with a standard computer (6 GB RAM), while in some cases, swapping do not allow us to reconstruct images with a cartesian matrix.

In order to compare the results obtained with iterative methods using polar matrix approach against FBP reconstructed images, the same set of CT data has been reconstructed with both methods.

The Feldkamp [1] algorithm has been implemented. Experimental measurements were binned, so that the projection bin and the reconstructed image voxel sizes were similar. The same binning was done for OSEM and FBP in order to 
compare results.

Although high resolution images with pixel sizes as small as $80 \mu \mathrm{m}$ can be achieved with the FBP, voxel sizes of $0.8 \mathrm{~mm}$ and $0.4 \mathrm{~mm}$ were considered in order to compare the reconstructed images.

The iterative method provides images with less noise than FBP in all RoIs, except that corresponding to Teflon (soft bone-like), see Tab. 2. In all soft tissues RoIs (fat/adipose tissue-like, soft tissue-like, and organs-like), noise values provided by OSEM were comparable to those provided by FBP, but the latter required an increase in the number of projections two to four times higher. Therefore, the iterative method allows a reduction of roughly $50 \%$ in the number of projections (and consequently the dose) needed to provide an image quality comparable to FBP.

We have found that the variation of $C R C$ with the number of projections is negligible, about $\pm 1 \%$, in both FBP and OSEM algorithms. However, depending on the RoI under analysis, $C R C$ values obtained with the OSEM in the soft bone and fat inserts are $95 \%$ of those measured with the FBP. The slower convergence speed of the OSEM in low-count regions [36], [37] leads to larger differences in the CRC values measured in the air region. Although being smaller than those measured with the FBP, CRC values obtained with the OSEM are above $80 \%$ in all cases.

Results in Fig. 10 confirm that useful images for medical applications in small animals using voxel sizes as big as $0.8 \mathrm{~mm}$ are possible when iterative methods are considered. FBP reconstruction requires voxel sizes smaller than $0.4 \mathrm{~mm}$ to provide useful images, therefore increasing their sizes.

\section{Preferred position for figure 11}

Figure 11 shows the same phantom rendered to an opaque volume Fig. 11(a) and semitransparent volume Fig. 11(b). These figures were obtained by render- 
ing the entire volume of Fig. 10(c).

\section{Conclusions}

In this work we have developed a 3D polar matrix for OSEM iterative image reconstruction method (or MLEM) which allows us to study 3D measurements in a standard computer (6 GB RAM) without losing quality or stability in the reconstructed images. Three different polar 2D matrices have been compared, the one offering us the better results has been extended to 3D.

It is worth pointing out that the proposed polar discretization reduced the computer storage requirements of the system matrix by a factor that equals the number of projections used in the tomographic system. As this number is usually close to 200 projections, this implies a huge reduction in storage requirements. As the computational complexity was significantly reduced, reconstructions of three dimensional (3D) CT images were permitted without losing in quality.

A major health concern today is related to the reduction of dose to the patient which means limiting either the X-ray source intensity or the number of projections [38], [39]. Iterative methods allow a high reductions dose [6], [9], due to the reduction in the number of projections necessary to reconstruct the image, but iterative methods have the disadvantage of the computational cost and the size of the system matrix used for the reconstruction. The proposed polar matrix allows us the use of the MLEM and/or OSEM algorithms with more detailed matrix (decreasing in the voxel size) with the same computer resources.

It should be pointed out that the proposed polar discretization can be easily implemented in large human CT, therefore allowing the use of iterative methods, such as MLEM and OSEM, as the time needed to reconstruct diagnostic images is compatible with time requirements of the clinical daily routine. 


\section{Conflict of interest statement}

None declared

\section{Acknowledgments}

This work was supported by the Spanish Plan Nacional de Investigación Científica, Desarrollo e Innovación Tecnológica (I+D+I) under Grant, FIS201021216-CO2-01, Valencian Local Government under Grant Nos. PROMETEO 2008/114 and APOSTD/2010/012. The authors would like to thank Brennan Holt for checking and correcting the text.

\section{References}

[1] L.A. Feldkamp, L.C. Davis and J.W. Kress, Practical cone-beam algorithm, J. Opt. Soc. Am. A 1 (1984) 612-619.

[2] L. Sheep and Y. Vardi, Maximum likelihood reconstruction for emission tomography, IEEE T. Med. Imaging 1 (1982) 113-122.

[3] H.M. Hudson and R.S. Larkin, Accelerated image reconstruction using ordered subsets of projection data, IEEE T. Med. Imaging 13 (1994) 601-609.

[4] E.Y. Sidky and X. Pan, Image reconstruction in circular cone-beam computed tomography by constrained, total-variation minimization, Phys. Med. Biol. 53 (2008)4777-4807.

[5] A. Del Guerra, N. Belcari, State-of-the-art of PET, SPECT and CT for small animal imaging, Nucl. Instr. and Meth. A 583 (2007) 119-124.

[6] C. Ghetti, O. Ortenzia and G. Serreli, CT iterative reconstruction in image space: A phantom study, Phys. Medica 28 (2012) 161-165. 
[7] F.A. Miéville, F. Gudinchet, F. Brunelle, F.O. Bochud and F.R. Verdun, Iterative reconstruction methods in two different MDCT scanners: Physical metrics and 4-alternative forced-choice detectability experiments-A phantom approach, Phys. Medica 29 (2013) 99-110.

[8] A. K. Hara, R.G. Paden, A.C. Silva, J.L. Kujak, H.J. Lawder and W. Pavlicek, Iterative reconstruction technique for reducing body radiation dose at CT: Feasibility study, Am. J. Roentgenol. 193 (2010) 764-771.

[9] M. Beister, D. Kolditz and W.A. Kalender, Iterative reconstruction methods in X-ray CT, Phys. Medica 28 (2012) 94-108.

[10] A. J. Reader, The promise of new PET image reconstruction, Phys. Medica $24(2008) 49-56$.

[11] K. S. Chuang, M.L. Jan, J. Wu, J.C. Lu, S. Chen, C.H. Hsu and Y.K. Fu, A maximum likelihood expectation maximization algorithm with thresholding. Comput. Med. Imag. Grap. 29(7) (2005) 571-578.

[12] C. Tsoumpas, F. Turkheirmer and K. Thielemans, Convergence properties of algorithms for direct parametric estimation of linear models in dynamic PET. IEEE Nuclear Science Symposium Conference Record (2007) 30343037.

[13] A. Gaitanis, G. Kontaxakis,G. Spyrou, G. Panayiotakis and G. Tzanakos, PET image reconstruction: A stopping rule for the MLEM algorithm bases on properties of the updating coefficients, Comput. Med. Imag. Grap. 34 (2010) 131-141.

[14] G.I. Angelis, K. Thielemans, A.C. Tziortzi, F. E. Tukheirmer and C. Tsoumpas, Convergence optimization of parametric MLEM reconstruction 
for estimation of Patlak plot parameters. Comput. Med. Imag. Grap. 35 (2011) 407-416.

[15] Z. Yu, J.B. Thibault, C.A. Bouman, K.D. Sauer, J. Hsieh, Fast modelbased X-ray CT reconstruction using spatially nonhomogeneous ICD optimization, IEEE T. Image Process.20(1) (2011) 161-175.

[16] J.E. Ortuño, J.L. Rubio, P. Guerra, G. Kontaxakis, M. Desco, J. Vaquero and A. Santos, Efficient methodology for 3D statistical reconstruction of high resolution coplanar PET/CT scanner, IEEE Nuclear Science Symposium Conference Record (2008) 5096-5100.

[17] A. Sen, H-H. Hsiung, M. Patel, B. Schueler, J.E. Holte and X. Hu, An exact technique for weighting function calculation in $3 \mathrm{D}$ cone beam recontruction, SPIE. 2432(1995) 616-626.

[18] W. Yao and K. Leszczynski, Analytically derived weighting factors for transmission tomography cone beam projections, Phys. Med. Biol. 54 (2009) 513-533.

[19] M. Hupfer, D. Kolditz, T. Nowak, F. Eisa, R. Brauweiler and W.A. Kalender, Dosimetry concepts for scanner quality assurance and tissue dose assessment in micro-CT, Med. Phys. 39(2) (2012) 658-670.

[20] A.P. Dempster, N.M. Laird, D.B. Rubin, Maximum Likelihood from Incomplete Data via the EM Algorithm, J. Roy. Stat. Soc. B Met. 39(1)(1977) $1-38$.

[21] W. Yang, X. Xu, K. Bi, S. Zeng, Q. Liu, and S. Chen, Adaptive region of interest method for analytical micro-CT reconstruction, J. X-Ray Sci. Technol. 19(1) (2011) 23-33. 
[22] R.M. Lewit, Multidimensional digital image representations using generalized Kaiser-Bessel window functions J. Opt. Soc. Am.7(10) (1990) 18341846.

[23] A. Yendiki and J.A. Fessler, A comparison of rotation-and blob-based system models for 3D SPECT with depth-dependent detector response, Phys. Med. Biol. 49 (2004) 2157-2168.

[24] A. Andreyev, M. Defrise and C. Vanhove, Pinhole SPECT reconstruction using blobs and resolution recovery, IEEE Nuclear science symposium conference record (2005) 2386-2390.

[25] Y.L. Hsieh, G.T. Gullberg, G.L. Zeng and R.H. Huesman, Image reconstruction using a generalized natural pixel basis, IEEE T. Nucl. Sci. 43(4) (1996) 2306-2319.

[26] C. Mora, M.J. Rodríguez-Alvarez and R.V. Romero, New pixellation scheme for $\mathrm{CT}$ algebraic reconstruction to exploit matrix symmetries, Comput. Math. Appl. 56 (2008) 715-726.

[27] G.H. Golub and C.H. Van Loan, Matrix Computations (3rd ed.), The Johns Hopkins University Press (1996) 201-202.

[28] M.J. Rodríguez-Alvarez, F. Sánchez, A. Soriano, A. Iborra, and C. Mora, Exploiting symmetries for weight matrix design in CT imaging, Mathl. Comput. Modelling 54 (2011) 1655-1664.

[29] P.M. Joseph, An improved algorithm for reprojection rays through pixel images, IEEE T. Med. Imaging MI-I 3 (1982) 192-196.

[30] http://www.gem-imaging.com/productos/albira/albira.php. Last accessed January-2013. 
[31] IEC Evaluation and routine testing in medical imaging departments-Part 3-5: Acceptance tests-Imaging performance of computed tomography X-ray equipment. (2004) 61223-3-5.

[32] IEC Evaluation and routine testing in medical imaging departments-Part 2-6: Constancy tests-Imaging performance of computed tomography X-ray equipment. (2006) 61223-2-6.

[33] International Commission on Radiation Units and Measurements (ICRU), Tissue substitutes in radiation dosimetry and measurement. (1989) Tech Rep 44.

[34] O. Nix, Exercises in PET image reconstruction. Molecular Imaging: Computer reconstruction and practice. Y. Lemoigne and A. Cainer (eds), Springer Science, (2008)131-44.

[35] A. Seret, The number of subsets required for OSEM reconstruction in nuclear cardiology, Eur. J. Nucl. Med. Mol. Imaging 33(2)(2006)231.

[36] S.H. Walrand, L.R. van Elmbt and S. Pauwels, A non-negative fast multiplicative algorithm in 3D scatter-compensated SPET reconstruction, Eur. J. Nuc. Med. 23 (11) (1996) 1521-1526.

[37] B.F. Hutton, H.M. Hudson and F.J. Beekman, A clinical perspective of accelerated statistical reconstruction, Eur. J. Nucl. Med. 24(7) (1997) 797 -808 .

[38] X. B. Dai, H.Z. Shu, L.M. Luo, G.N Han and J.L. Coatrieux, Reconstruction of tomographic images from limited range projections using discrete Radon transform and Tchebichef moments, Pattern. Recogn. 43 (2010) 1152-1164. 
[39] F. Zarb, L. Rainford, M.F. McEntee, Image quality assessment tools for optimization of CT images, Radiography 16(2) (2010) 147-153. 
Table 1: Comparison between 3D cartesian matrix and 3D polar matrix.

\begin{tabular}{|c|c|c|c|c|c|c|c|c|}
\hline \multirow{3}{*}{$\begin{array}{r}\mathrm{N}^{o} \text { voxels } \\
\text { Voxel size }\left(\mathrm{mm}^{3}\right) \\
\end{array}$} & \multicolumn{4}{|c|}{ cartesian matrix } & \multicolumn{4}{|c|}{ polar matrix } \\
\hline & \multirow{2}{*}{\multicolumn{2}{|c|}{$\begin{array}{c}96 \times 96 \times 88 \\
0.8 \times 0.8 \times 0.8\end{array}$}} & \multirow{2}{*}{\multicolumn{2}{|c|}{$\begin{array}{c}192 \times 192 \times 175 \\
0.4 \times 0.4 \times 0.4\end{array}$}} & \multirow{2}{*}{\multicolumn{2}{|c|}{$\begin{array}{l}102 \times 102 \times 88 \\
0.8 \times 0.8 \times 0.8\end{array}$}} & \multirow{2}{*}{\multicolumn{2}{|c|}{$\begin{array}{c}204 \times 204 \times 175 \\
0.4 \times 0.4 \times 0.4\end{array}$}} \\
\hline & & & & & & & & \\
\hline $\mathrm{N}^{O}$ projections & 100 & 200 & 100 & 200 & 100 & 200 & 100 & 200 \\
\hline Size & $1.2 \mathrm{~GB}$ & $2.5 \mathrm{~GB}$ & $3.9 \mathrm{~GB}$ & $8.5 \mathrm{~GB}$ & $60 \mathrm{MB}$ & $60 \mathrm{MB}$ & $224 \mathrm{MB}$ & $224 \mathrm{MB}$ \\
\hline Matrix generation & $88 \mathrm{~s}$ & $190 \mathrm{~s}$ & $271 \mathrm{~s}$ & $630 \mathrm{~s}$ & $5 \mathrm{~s}$ & $5 \mathrm{~s}$ & $12 \mathrm{~s}$ & $12 \mathrm{~s}$ \\
\hline Iteration time ${ }^{1}$ & $1.4 \mathrm{~s}$ & $3.5 \mathrm{~s}$ & $8.5 \mathrm{~s}$ & -2 & $1.8 \mathrm{~s}$ & $3.9 \mathrm{~s}$ & $7.1 \mathrm{~s}$ & $14.9 \mathrm{~s}$ \\
\hline
\end{tabular}

$\left(^{1}\right)$ (40 for MLEM and 5 for OSEM) iterations are used to reconstruct images of this paper.

$\left({ }^{2}\right)$ Iteration time was higher than 30 minutes due to swapping.

Table 2: Comparison of the dependence with the number of projections of the $C V$ (in \%) measured in each RoI (see Fig. 4). 20 subsets and 5 iterations were used for OSEM recontructions.

\begin{tabular}{|c|c|c|c|c|c|c|}
\hline & & \multicolumn{5}{|c|}{ Num Projections } \\
\hline & & 400 & 200 & 100 & 80 & 50 \\
\hline \multirow{2}{*}{ Air } & OSEM & 6.2 & 6.4 & 7 & 6 & 11.3 \\
\hline & FBP & 21 & 28 & 38 & 46 & 58 \\
\hline \multirow{2}{*}{ Fat/adipose tissue-like } & OSEM & 1.5 & 1.8 & 2.6 & 3 & 4.3 \\
\hline & FBP & 2.4 & 3.2 & 4.7 & 5.8 & 7.6 \\
\hline \multirow{2}{*}{ Soft tissue-like } & OSEM & 1.4 & 1.8 & 2.5 & 2.7 & 3.4 \\
\hline & FBP & 1.9 & 2.6 & 3.6 & 4.2 & 5.5 \\
\hline \multirow{2}{*}{ Organs-like } & OSEM & 1.2 & 1.6 & 2.3 & 2.5 & 3.2 \\
\hline & FBP & 1.5 & 2 & 3 & 3.6 & 4.9 \\
\hline \multirow{2}{*}{ Soft bone-like } & OSEM & 1.5 & 1.8 & 2.3 & 2.5 & 3.1 \\
\hline & FBP & 1.2 & 1.4 & 1.9 & 2 & 2.6 \\
\hline
\end{tabular}

Table 3: Comparison of the $C R C$ (in \%) measured in each RoI (see Fig. 4). 20 subsets and 5 iterations were used for OSEM reconstructions.

\begin{tabular}{rcc} 
& OSEM & FBP \\
\hline Air & 83 & 91 \\
Fat/adipose tissue-like & 86 & 92 \\
Soft bone-like & 82 & 85 \\
\hline
\end{tabular}




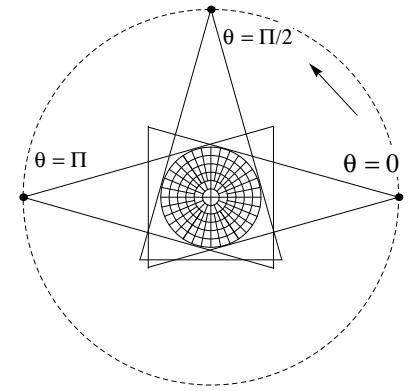

(a)

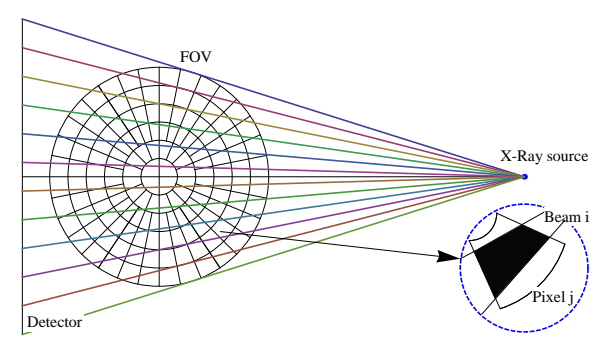

(b)

Figure 1: (a) Symmetries for a CT scanner with a polar pixellation. (b) Diagram of pixel weight calculation.

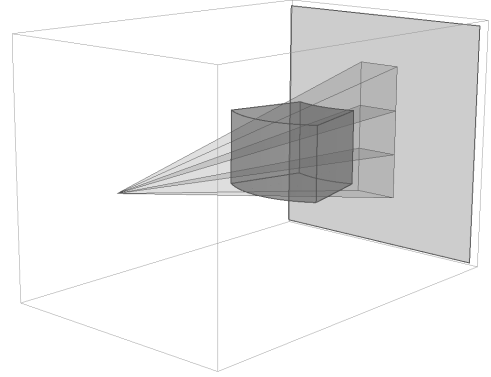

Figure 2: 3D Voxel scanned by three beams. Figure is not to scale.

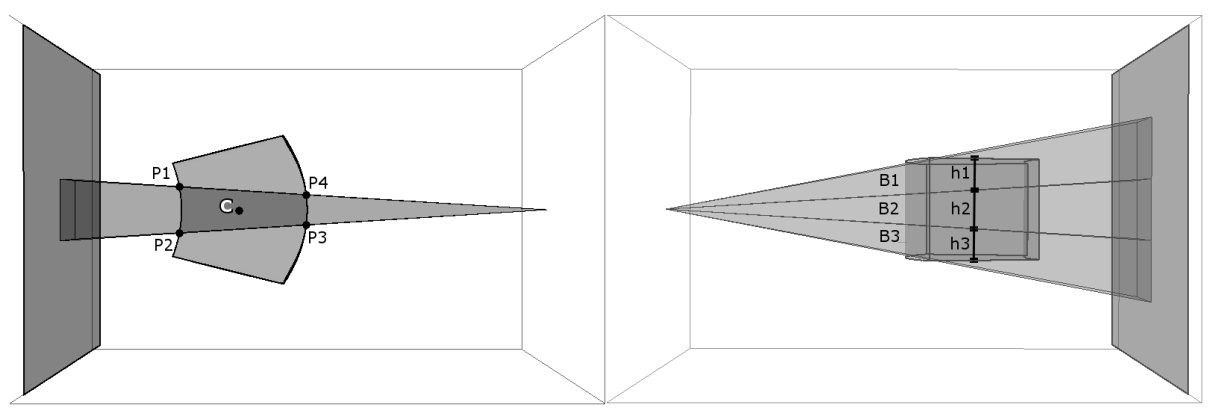

(a) Top view

(b) Lateral view

Figure 3: Voxel/beam intersection. Figures are not to scale. 


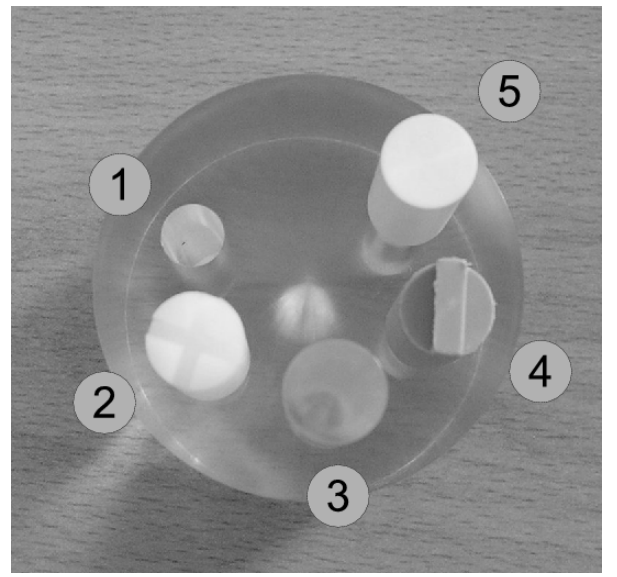

Figure 4: Phantom with material inserts. The numbers on the image correspond to: 1 Air, 2 PFTE, 3 PMMA, 4 PE and 5 POM. 


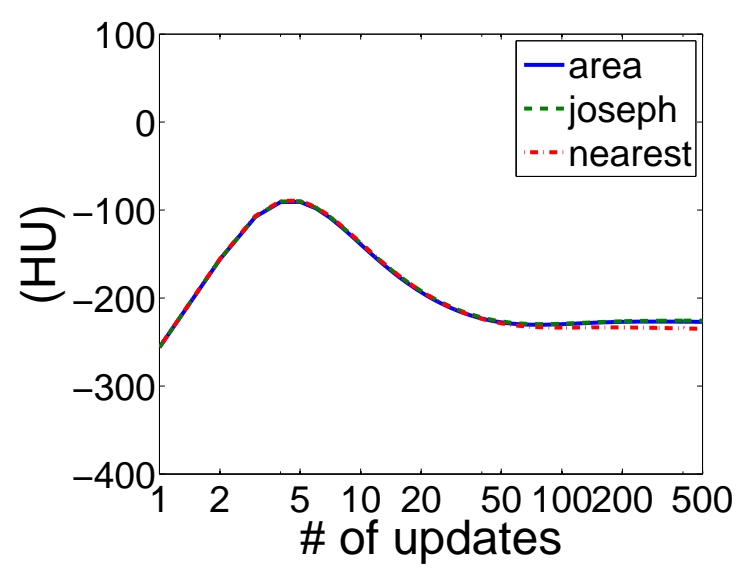

(a) 1 subset

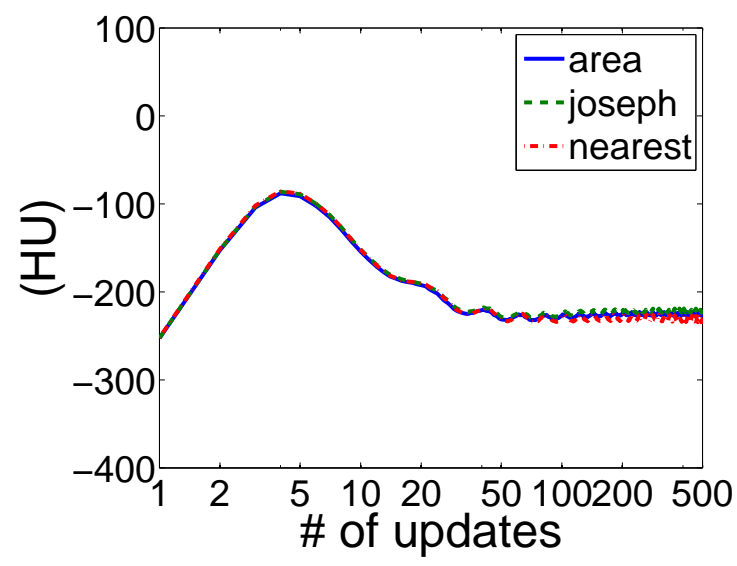

(c) 20 subsets

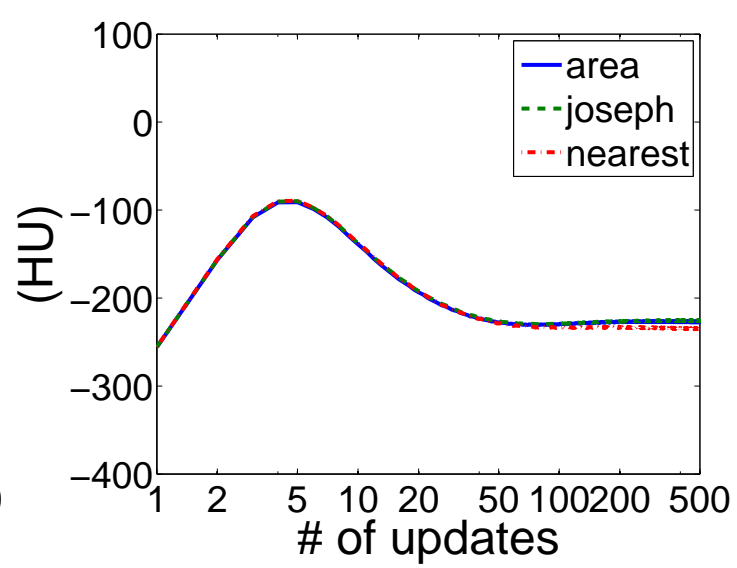

(b) 10 subsets

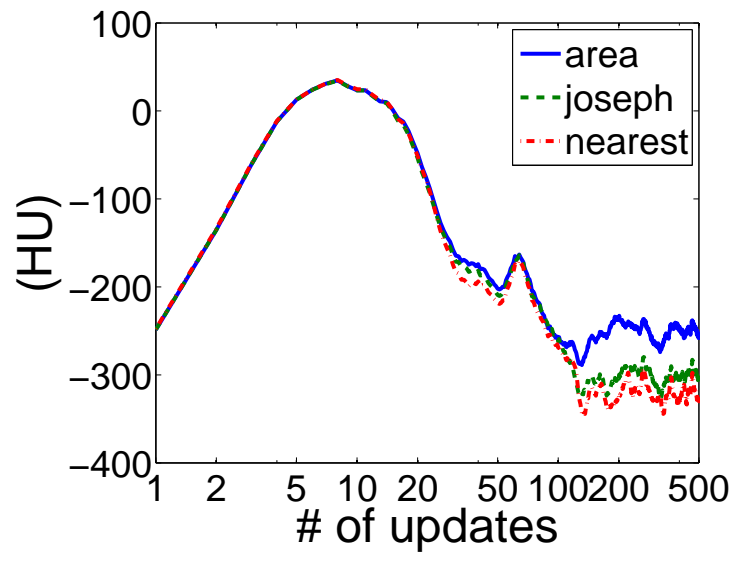

(d) 100 subsets

Figure 5: CT number (in Hounsfield Units, HU) of reconstructed images for Polyethylene (PE) RoI as a function of the number of updates. 


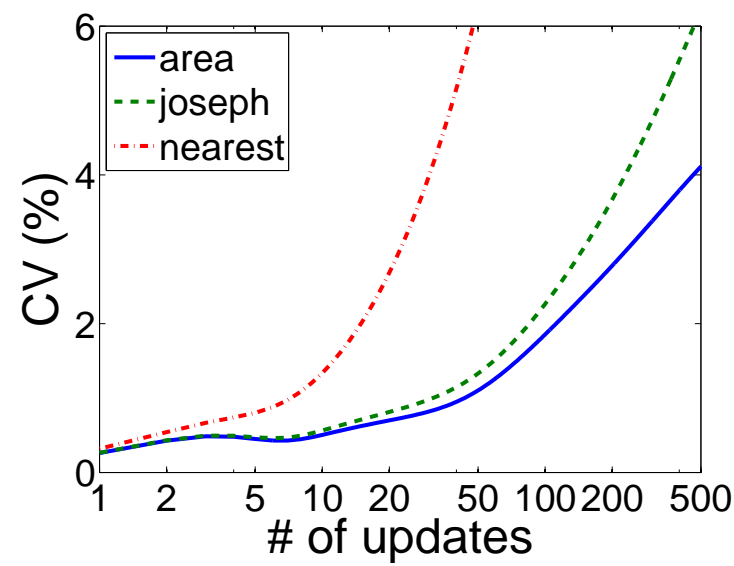

(a) 1 subset

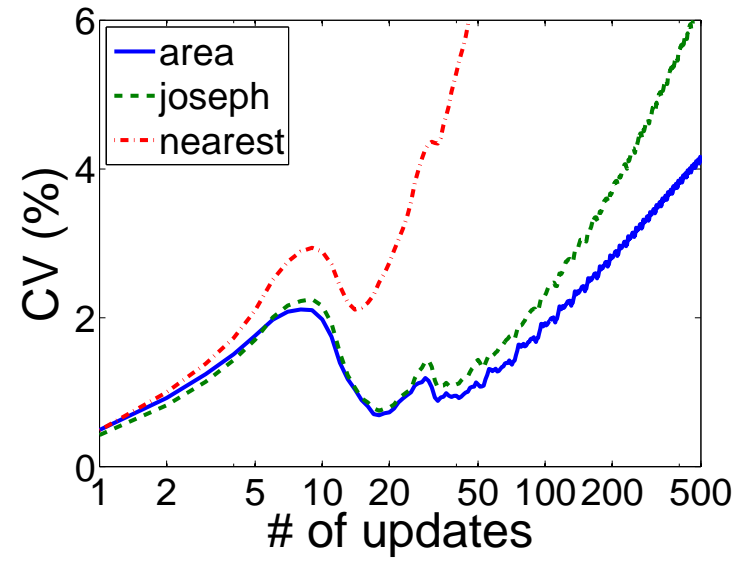

(c) 20 subsets

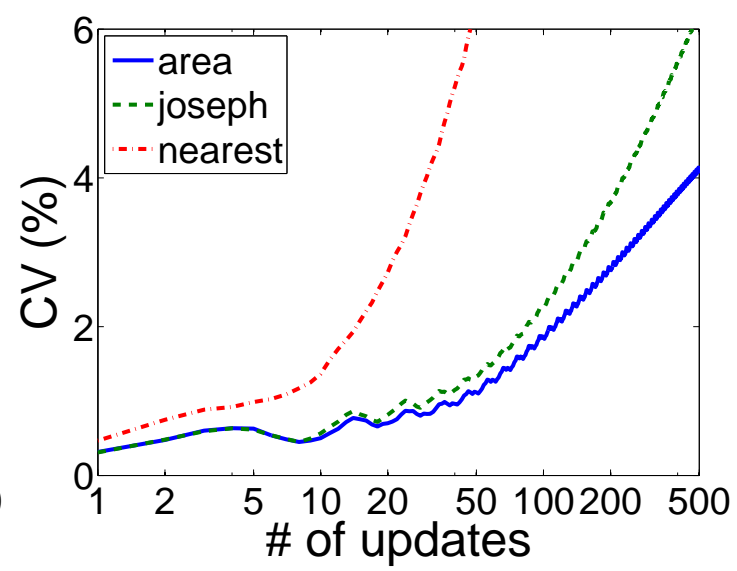

(b) 10 subsets

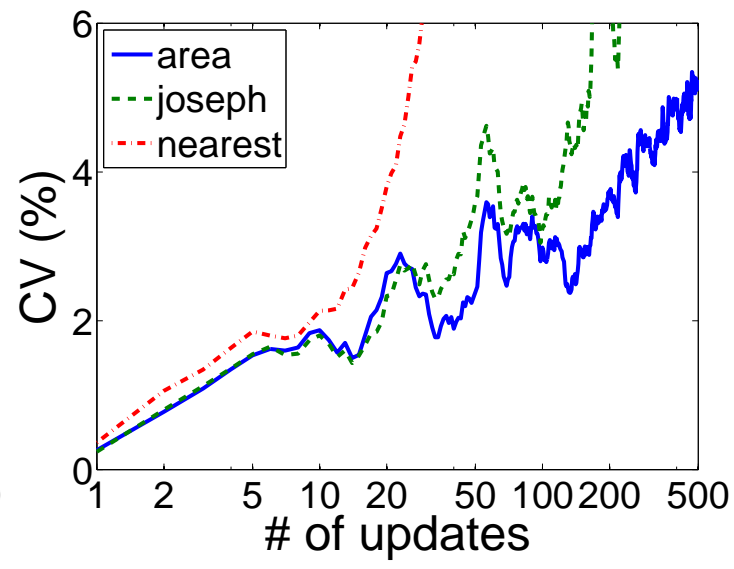

(d) 100 subsets

Figure 6: $C V$ of reconstructed images for Polyethylene (PE) RoI as a function of the number of updates. 


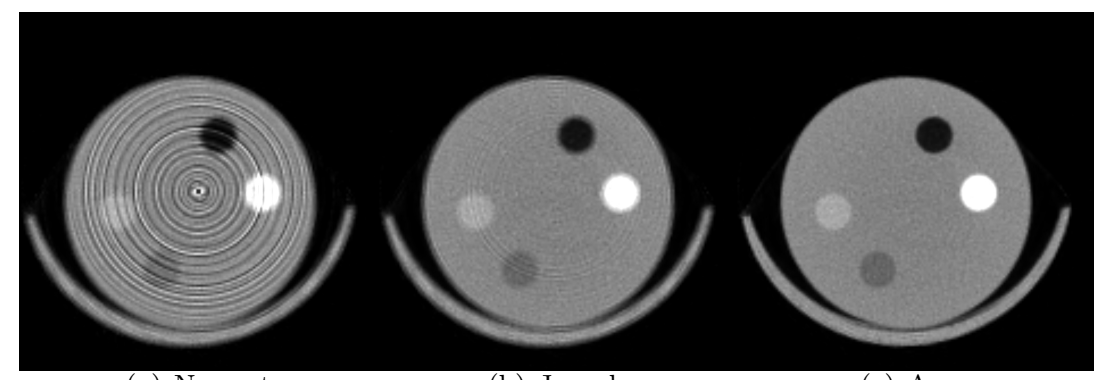

(a) Nearest

(b) Joseph

(c) Area

Figure 7: 2D reconstructed images. 


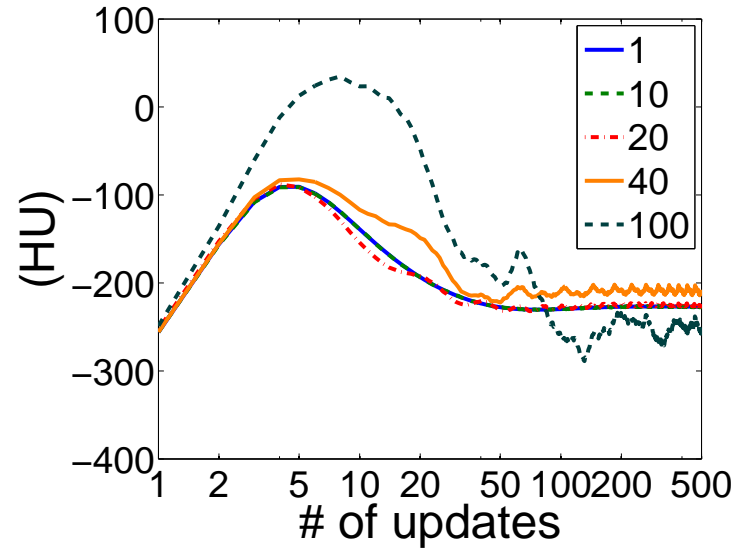

(a) CT number

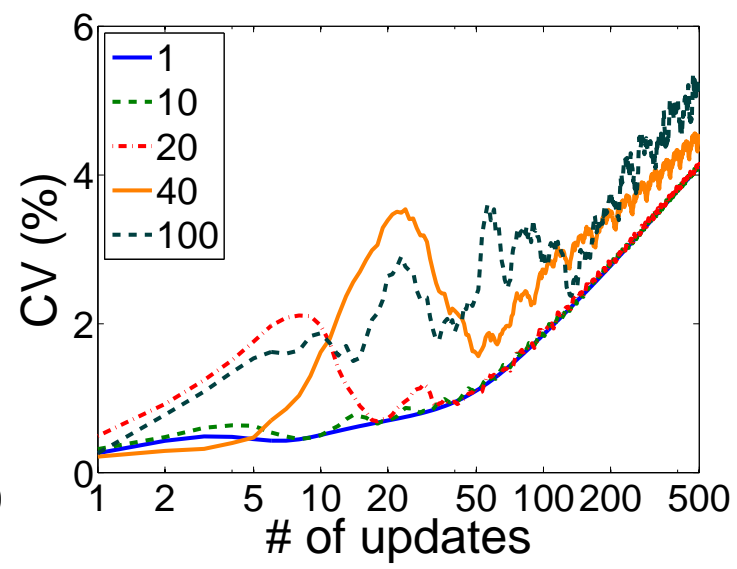

(b) $\mathrm{CV}$

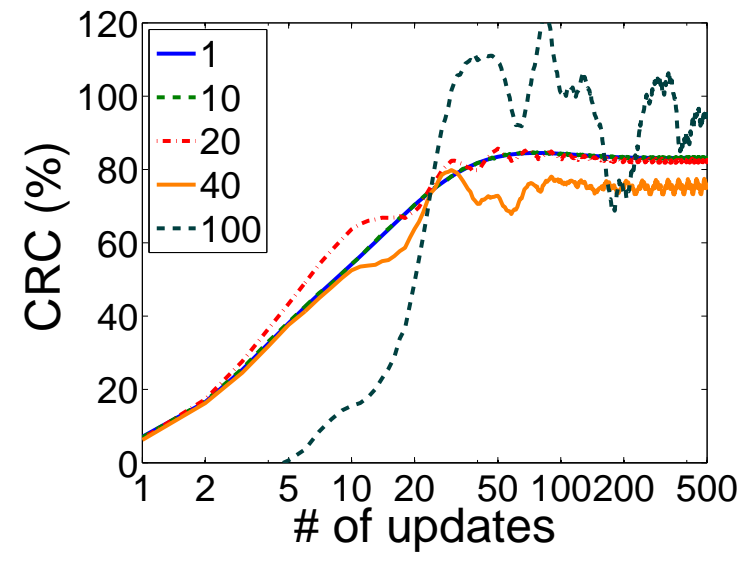

(c) $\mathrm{CRC}$

Figure 8: Results obtained for 2D sector matrix as a function of update number for 1, 10, 20, 40 and 100 subsets for Polyethylene (PE) RoI. 


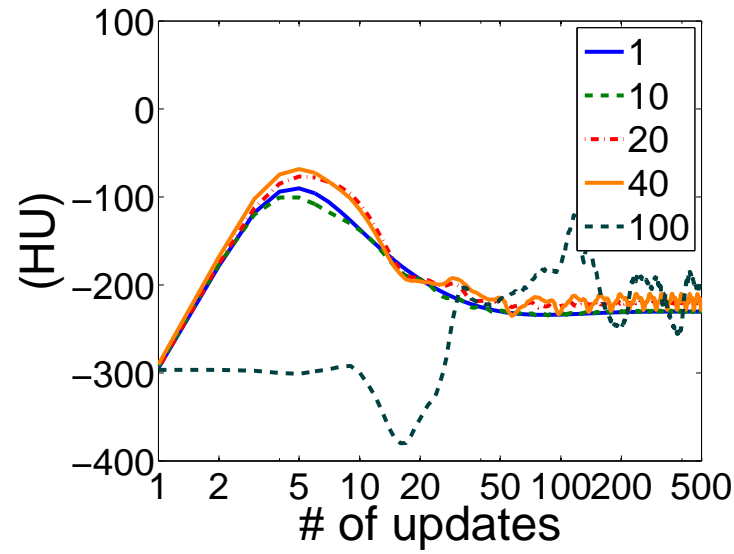

(a) CT number

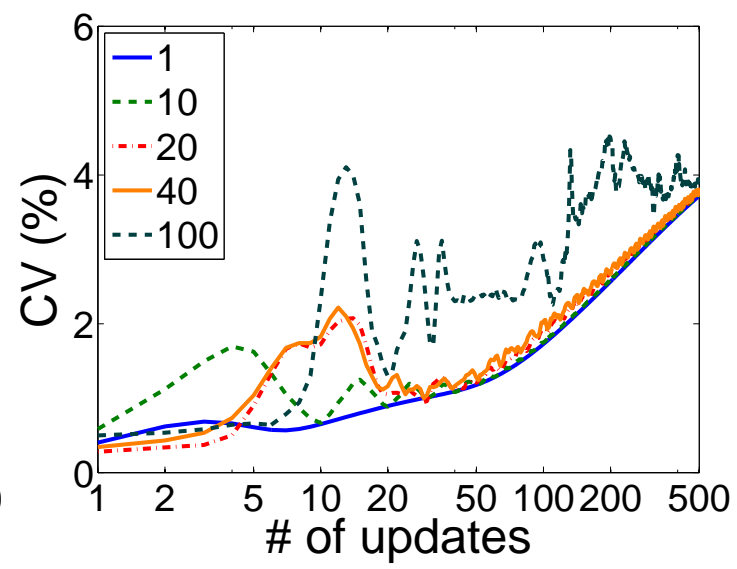

(b) $\mathrm{CV}$

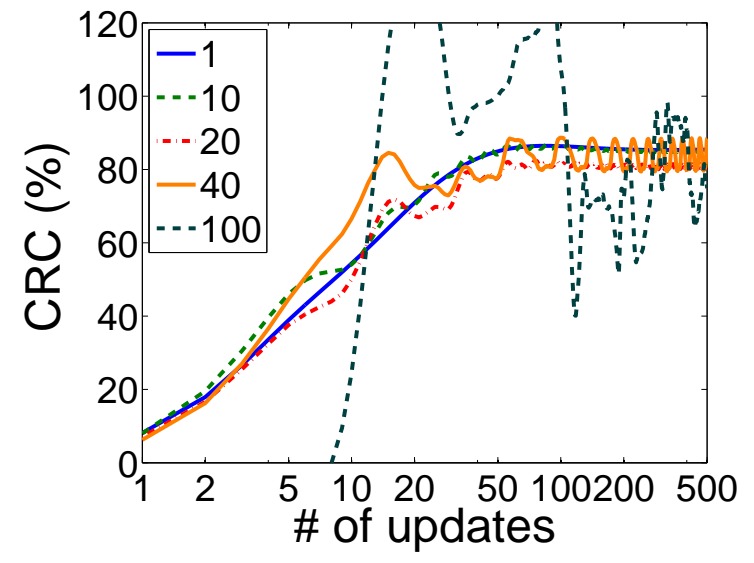

(c) $\mathrm{CRC}$

Figure 9: Results obtained for 3D sector matrix for Polyethylene (PE) RoI as a function of update number for 1, 10, 20, 40 and 100 subsets . 


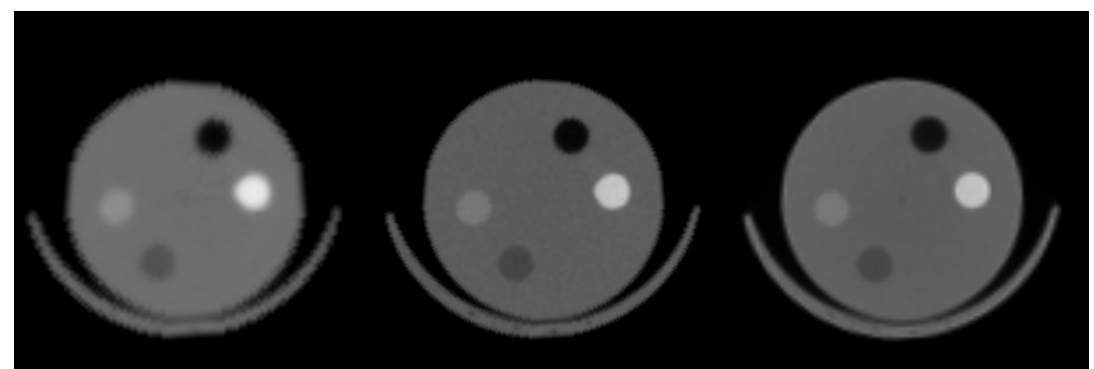

(a) FBP $0.8 \mathrm{~mm}$

(b) FBP $0.4 \mathrm{~mm}$

(c) Iterative $0.8 \mathrm{~mm}$

Figure 10: Comparison of transversal 3D images reconstructed with OSEM and FBP with several voxel sizes.

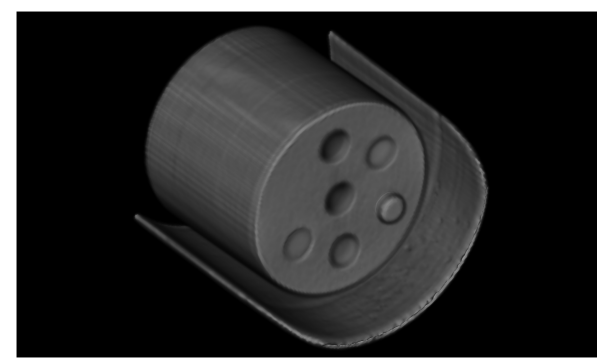

(a)

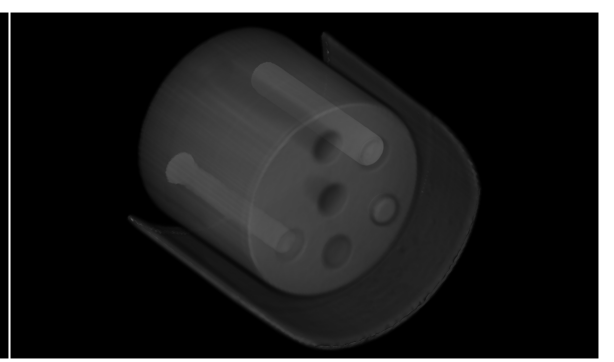

(b)

Figure 11: Phantom reconstructed with OSEM polar matrix rendered to (a) opaque volume and (b) semitransparent volume. 